\title{
Pilot, Randomized, Blinded, Placebo-Controlled Trial Investigating the Correlation Between Acid Control and Heartburn Relief with 14 Days of Esomeprazole Treatment
}

Philip B. Miner · David A. Johnson · Philip O. Katz · Jing Li ·

Sergio C. Gatoulis · Charles Pollack

Received: June 8, 2018 / Published online: September 25, 2018

(C) The Author(s) 2018

\section{ABSTRACT}

Introduction: Reflux symptoms are frequently associated with esophageal acid exposure. However, other potential causes unrelated to acid secretion are possible, and the relationship between acid control and symptomatic improvement remains unclear. This study investigated the correlation between individual intragastric $\mathrm{pH}$ control and heartburn relief among subjects with frequent heartburn who

Enhanced digital features To view enhanced digital features for this article go to https://doi.org/10.6084/ m9.figshare.7017266.

P. B. Miner $(\bowtie)$

Oklahoma Foundation for Digestive Research,

Oklahoma City, OK, USA

e-mail: philip-miner@ofdr.com

D. A. Johnson

Eastern Virginia Medical School, Norfolk, VA, USA

P. O. Katz

Weill Cornell Medicine, New York, NY, USA

J. Li

Biostatistics, Pfizer Consumer Healthcare, Madison,

NJ, USA

S. C. Gatoulis

Global Clinical Research, Pfizer Consumer

Healthcare, Madison, NJ, USA

C. Pollack

Global R\&D, Pfizer Consumer Healthcare, Madison, NJ, USA are likely to self-treat with over-the-counter (OTC) medications. We hypothesized that improved acid control would provide greater symptomatic improvement among individuals representative of an OTC population.

Methods: This phase 4, single-center, randomized, double-blind, placebo-controlled study was conducted in subjects without diagnosed gastroesophageal reflux disease or other gastrointestinal conditions who were experiencing frequent heartburn ( $\geq 3$ episodes/week; $\geq 2$ nighttime episodes/week over past 30 days) that was responsive to treatment. Subjects entered a 7-day run-in phase, received placebo BID (before breakfast and dinner), and completed symptom diaries. During the treatment phase, subjects received esomeprazole $20 \mathrm{mg}$ BID, esomeprazole $20 \mathrm{mg}$ then placebo, or placebo BID. Subjects underwent 24-h intragastric $\mathrm{pH}$ monitoring at baseline and day 14 and completed daily symptom diaries.

Results: In the per-protocol population $(n=39)$, mean (SD) change from baseline in percentage of time with intragastric $\mathrm{pH}>4$ was $58.7 \%( \pm 26.4 \%)$ versus $41.0 \%( \pm 30.4 \%)$ for those who did and did not achieve 24-h heartburn relief. Significant correlations were observed between change in percentage of time with intragastric $\mathrm{pH}>4$ and 24-h heartburn relief (OR 1.028; 95\% CI 1.001, 1.055; $P=0.0442)$ and complete resolution (OR 1.034; 95\% CI 1.003, 1.065; $P=0.0301)$. 
Conclusions: Individuals with greater improvements in duration of intragastric acid suppression had an increased likelihood of achieving heartburn relief and resolution. These results indicate that individuals not adequately controlling their intragastric $\mathrm{pH}$ may require an escalation in dose of their acid-suppressive therapy, assessment with $24-\mathrm{h} \mathrm{pH}$ monitoring, or a change in treatment regimen to address non-reflux-related etiologies of their heartburn.

Trial Registration: ClinicalTrials.gov identifier: NCT02708355.

Funding: Pfizer Consumer Healthcare, Madison, NJ, USA.

Plain Language Summary: Plain language summary available for this article.

Keywords: Esomeprazole; Frequent heartburn; Gastroenterology; Intragastric $\mathrm{pH}$

\section{PLAIN LANGUAGE SUMMARY}

In this study, subjects who did not have a diagnosis of gastroesophageal reflux disease, or GERD, but who were experiencing frequent symptomatic heartburn with no diagnostic medical evaluation were randomized to receive either placebo or esomeprazole $20 \mathrm{mg}$ once or twice a day. During the 14-day treatment period, subjects received either esomeprazole $20 \mathrm{mg}$ twice a day, esomeprazole $20 \mathrm{mg}$ and then placebo, or placebo twice a day and completed daily symptom diaries. Subjects' 24-h intragastric $\mathrm{pH}$ was monitored at baseline and on day 14 of the treatment period, using the standard definition of gastric acidity (i.e., $\mathrm{pH}>4$ ). The relationship between the change in the percentage of time from baseline to day 14 with 24-h $\mathrm{pH}>4$, heartburn relief, and complete heartburn resolution was evaluated.

Significant associations were observed between the changes from baseline to day 14 in the individuals' percentage of time with intragastric $\mathrm{pH}>4,24$-h heartburn relief, and complete heartburn resolution.

We concluded that the likelihood of an individual experiencing heartburn relief or resolution improved as the control over intragastric $\mathrm{pH}$ increased. These effects have only previously been observed using populationlevel means, so this is the first time this relationship has been observed using individual data. As expected, esomeprazole treatment increased the likelihood of experiencing treatment response, but symptomatic response was also observed with placebo and was dependent on the individual degree of $\mathrm{pH}$ control. These results suggest that individualized assessments can provide guidance in making treatment decisions for individuals not achieving adequate $\mathrm{pH}$ control.

\section{INTRODUCTION}

The role of esophageal acid exposure in reflux symptoms (i.e., heartburn, acid regurgitation) is unequivocal and provides the rationale for using acid-suppressive therapy [1]. In fact, the American College of Gastroenterology recommends empirical use of acid-suppressive therapy to manage reflux symptoms prior to conducting diagnostic investigations [2]. The degree of mucosal injury in erosive esophagitis (EE) is correlated with the amount of time with intragastric $\mathrm{pH}<4$ [3]. As a result, the percentage of time in a $24-\mathrm{h}$ period when intragastric $\mathrm{pH}$ is $>4$ is an important metric for assessing the pharmacodynamic effects of acid-suppressive therapies [4-8].

Acid-suppressive therapies can significantly increase the percentage of time with intragastric $\mathrm{pH}>4$ within 7 days of initiation $(P<0.01)$ $[4,5]$. Sustained suppression of gastric acid secretion with proton-pump inhibitors (PPI) or histamine receptor 2 antagonists $\left(\mathrm{H}_{2} \mathrm{RA}\right)$ plays a role in treating acid-related diseases $[1,9]$. PPIs are commonly used in both over-the-counter (OTC) and prescription settings and block the final step of gastric acid production, inhibiting both basal and stimulated acid secretion $[1,10]$. In one study, healing rates in EE were positively correlated with percentage of time with intragastric $\mathrm{pH}>4$ following 4 weeks of esomeprazole treatment [11]. This study also suggested that greater intragastric acid control lowered daytime and nighttime heartburn and acid regurgitation [11]. Extrapolation of the relationship between acid suppression and 
heartburn relief in individuals with non-erosive reflux is accepted; however, the relationship between the degree of acid suppression and symptom relief on an individual level has not been investigated.

The current pilot proof-of-concept study was designed to explore the relationship between intragastric acid control and reflux symptoms by investigating associations between change from baseline in time with intragastric $\mathrm{pH}>4$ and 24-h heartburn relief using individual-subject data. We hypothesized that individuals with frequent heartburn would experience greater symptomatic improvement with improved acid control (i.e., greater reductions in $\mathrm{pH}$ ). Because responses to medicine vary from person to person, individual titration by the consumer may be necessary to optimize outcomes among those who do not achieve complete resolution of heartburn with PPI treatment. Thus, exploring these issues in the context of individuals who may self-treat their reflux symptoms using an OTC PPI will provide additional insights that may potentially guide alternative treatment options for nonresponders.

\section{METHODS}

\section{Study Design}

This phase 4, single-center, randomized, double-blind, placebo-controlled, parallel-group pilot study (conducted between January 22, 2016, and April 3, 2016) included a 1-week single-blind placebo run-in phase followed by randomization into a 2-week double-blind treatment phase (Fig. 1). The run-in phase (days -8 to -1 ) was used to wash out any previous $\mathrm{H}_{2} \mathrm{RA}$ and/or PPI therapy, confirm the incidence of symptoms reported at screening, and determine whether subjects could accurately complete daily diaries. Subjects completed a daily diary to document occurrences of heartburn, acid regurgitation, and epigastric pain during the previous $24-\mathrm{h}$ period. The scale evaluated the presence and absence of symptoms and severity $(0=$ none; $2=$ severe $)$ but not the number of events. Subjects began taking placebo twice daily (before breakfast and dinner) on day -8 and began reporting symptoms on day -7 to -1 . On day -1 , subjects also completed the Quality of Life in Reflux and Dyspepsia (QOLRAD) questionnaire.

Following the run-in, subjects returned for potential baseline $\mathrm{pH}$ monitoring. Eligible subjects were required to report taking $\geq 80 \%$ of placebo doses ( $\leq 2$ missed doses) and experiencing $\geq 1$ heartburn episode during three separate 24-h periods; two episodes were required to occur during the night (i.e., nighttime heartburn; occurring during the time after consuming the evening meal until getting up in the morning to start daily activities) in two separate 24 -h periods. Eligible subjects were required to be compliant in reporting symptoms via a daily diary for $\geq 6$ days of the 7-day run-in. Subjects who met randomization criteria initiated 24-h catheter-based intragastric and esophageal $\mathrm{pH}$ monitoring. Subjects remained at the center for $\sim 11 \mathrm{~h}$ then returned the next day (day 1) to receive their first dose of study medication.

Treatment assignment was determined by a computer-generated randomization schedule generated and maintained by Pfizer Global Randomization Operations, and third-party personnel dispensed study medication. Only these individuals had access to the randomization schedule and dispensing records.

This study complied with the ethical principles of the Declaration of Helsinki and all International Conference for Harmonisation Good Clinical Practice Guidelines. The final protocol, any amendments, and informed consent forms were reviewed and approved on December 9, 2015, by an independent institutional review board at the investigational center (Oklahoma Foundation for Digestive Research; Oklahoma City, OK, USA). Informed consent was obtained from all individual participants included in the study. This study is registered with ClinicalTrials.gov (NCT02708355).

Eligible subjects were randomized to one of three 14-day treatment arms (2:2:1 ratio): esomeprazole $20 \mathrm{mg}$ [administered as $22.3 \mathrm{mg}$ esomeprazole magnesium trihydrate $\left(\right.$ Nexium $^{\circledR}$ 24HR; Pfizer Consumer Healthcare, Madison, NJ, USA)] before both breakfast and dinner; 


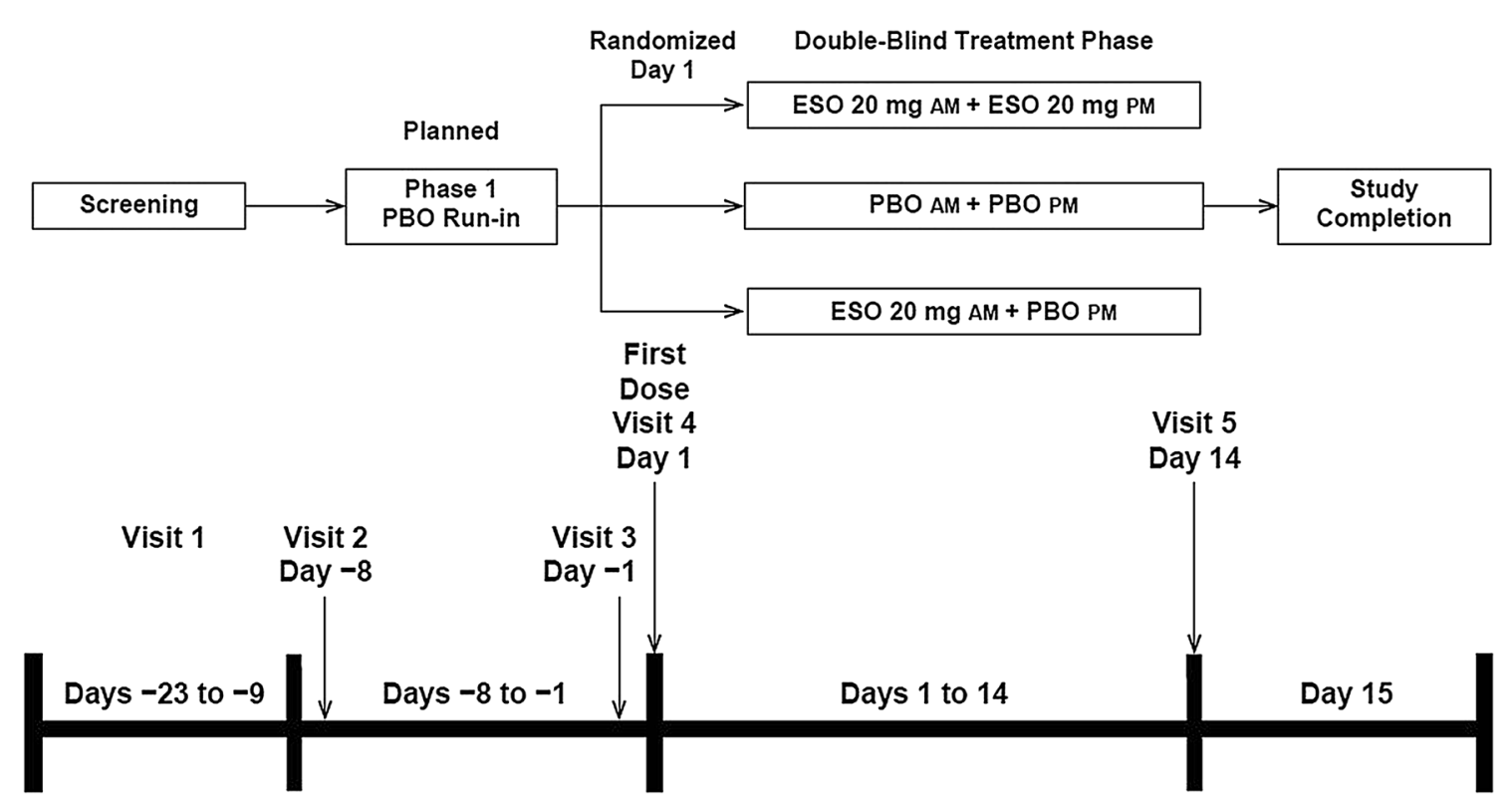

Fig. 1 Schematic of study design. ESO esomeprazole, PBO placebo

esomeprazole $20 \mathrm{mg}$ before breakfast/placebo before dinner; and placebo/placebo (both before breakfast and dinner). For non-pH-monitoring days, subjects were instructed to take the first and second daily doses 30-60 min before breakfast and dinner, respectively, to reflect product labeling for OTC PPIs that are used to self-treat heartburn and allow for variability in meal timing when subjects were away from the study center. When possible, breakfast and dinner were separated by $\geq 10 \mathrm{~h}$ (e.g., breakfast, 8:00 AM; dinner, 6:00 PM). A chewable antacid containing aluminum hydroxide $200 \mathrm{mg}$, magnesium hydroxide $200 \mathrm{mg}$, and simethicone 25 mg (Mintox ${ }^{\mathrm{TM}}$ Plus; Major Pharmaceuticals, Livonia, MI, USA) was provided as rescue medication. Subjects chewed one tablet as needed and repeated hourly if symptoms returned $(\leq 6$ tablets/24-h period and $\leq 28$ tablets/7-day period). No rescue medications were allowed during the $24-\mathrm{h} \mathrm{pH}$ monitoring period on day -1 or 14 .

Subject diaries recorded the incidence of daily heartburn, acid regurgitation, and epigastric pain in the morning for the previous $24-\mathrm{h}$ period throughout the 14-day treatment period. QOLRAD assessments, which described subjects' experiences over the previous 7 days, were conducted on day 14. After completing day-14 24-h $\mathrm{pH}$ monitoring, subjects returned to the center for final assessments and catheter removal.

\section{Study Population}

Subjects were recruited from the study site's database and through advertising. Inclusion criteria included male and nonpregnant, nonlactating female subjects, 22-65 years of age, with $17.5-45.0 \mathrm{~kg} / \mathrm{m}^{2}$ body mass index (BMI) and total body weight $>50 \mathrm{~kg}(110 \mathrm{lbs})$, heartburn averaging $\geq 3$ episodes/week, including $\geq 2$ episodes/week of nighttime heartburn, over the past 30 days, and confirmed heartburn, acid regurgitation, or epigastric pain histories for $\geq 3$ months that responded to antacids, $\mathrm{H}_{2} \mathrm{RAs}$, and/or PPIs. To reflect a typical population that would self-treat their heartburn with an OTC product, subjects did not undergo diagnostic testing and those with evidence/history of clinically significant diseases including gastrointestinal conditions (other than frequent heartburn), histories of endoscopically verified EE (GERD), and need for continuous $\mathrm{H}_{2} \mathrm{RAs}$, PPIs, gastric prokinetic drugs, or antacids for any indication (e.g., subjects with long-term prescription therapy) were not enrolled. The use 
of empiric PPI therapy to initially treat heartburn is recommended and common clinical practice [2]. Male and female subjects of childbearing potential were required to use highly effective contraceptives throughout the study and for $\geq 28$ days after the last treatment dose. Women of nonchildbearing potential must have met $\geq 1$ of the following criteria: confirmed postmenopausal status, documented hysterectomy and/or bilateral oophorectomy, or medically confirmed ovarian failure.

\section{Intragastric and Esophageal $\mathrm{pH}$ Monitoring}

To monitor intragastric and esophageal $\mathrm{pH}$, a catheter probe (ComforTec Z/pH Probe; Sandhill Scientific Inc, Highlands Ranch, CO, USA) was inserted transnasally. A distal esophageal sensor was placed $\sim 5 \mathrm{~cm}$ above the manometrically located proximal border of the lower esophageal sphincter; another was placed intragastrically $\sim 10 \mathrm{~cm}$ below the lower esophageal sphincter. The percentage of time with intragastric $\mathrm{pH}>4$ and esophageal $\mathrm{pH}<4$ and median intragastric and esophageal $\mathrm{pH}$ over $24 \mathrm{~h}$ on days -1 and 14 were evaluated. On $\mathrm{pH}$ monitoring days, standardized meals were provided to limit the effects of food choices. Breakfast was served $60 \mathrm{~min}$ after starting $\mathrm{pH}$ recording of day -1 , and, on day 14 , breakfast was served $30 \mathrm{~min}$ after morning dosing. On day 14 , lunch, snack, and dinner were served at 4,7 , and $10 \mathrm{~h}$, respectively, after morning dosing; evening doses were administered $9.5 \mathrm{~h}$ after morning dosing. After dinner, subjects were discharged with the nasal catheter in place and returned home with the $\mathrm{pH}$ monitoring data logger, a daily symptom diary, and rescue medication.

\section{Efficacy Evaluations}

The primary evaluation was the association between change in percentage of a 24 -h day with intragastric $\mathrm{pH}>4$ and 24-h heartburn relief. Relief of 24-h heartburn was defined as a daily diary response of " 0 " to the question, "Over the last $24 \mathrm{~h}$ (yesterday/last night), what was the severity of your most intense episode of heartburn?" on $\geq 6$ of subjects' last 7 consecutive treatment days, allowing for 1 day with a maximum severity of "2." All efficacy parameters are described in Table 1.

In addition to objective parameters, the impact on quality of life was evaluated using the QOLRAD [12], which measures the impact on emotional distress, sleep disturbance, food and drink problems, physical and social functioning, and vitality on a 7-point Likert scale. Lower scores indicate more severe impact on daily functioning. Assessments were conducted on days -1 and 14 and focused on the previous 7 days. Mean scores on each domain were used to calculate improvement from days -1 to 14 .

\section{Safety}

The investigator recorded any observed or volunteered adverse events (AEs), including severity (mild, moderate, or severe) and investigator's opinion of the relationship to treatment. The investigator recorded clinically significant changes in physical examination and abnormal objective test findings (e.g., electrocardiogram, laboratory) as AEs.

\section{Statistics}

The per-protocol (PP) analysis set was the primary population of interest and included only evaluable subjects, defined as those who completed the 14-day treatment phase, provided valid data for day $14 \mathrm{pH}$ monitoring, and completed $\geq 5$ days of diary entries for days -7 to -1 and 8-14. All analyses except for safety assessments were conducted with this population. To further explore the relationship between change from baseline in percentage of time with intragastric $\mathrm{pH}>4$ and heartburn relief and complete resolution, the full analysis set (FAS) was also analyzed. The FAS population was defined as all randomized subjects who provided valid day $-1 \mathrm{pH}$ monitoring data and took $\geq 1$ dose of randomized study medication. Approximately 100 subjects were planned to be enrolled and approximately 75 subjects to be randomized into the treatment phase, so that 
Table 1 Summary and definitions of efficacy parameters

Parameter Definition

Relief

Heartburn

24-h

Nighttime $^{a}$

Acid regurgitation

Epigastric pain

Complete resolution

Heartburn

24-h

Nighttime $^{a}$

Acid regurgitation

Epigastric pain

Improvement in frequency

Heartburn

24-h

Nighttime $^{a}$

Improvement in average number of events

Heartburn

Acid regurgitation

Epigastric pain

Improvement in severity

Heartburn

24-h

Nighttime ${ }^{a}$

24-h period with no events

Heartburn

24-h

Acid regurgitation

Epigastric pain

${ }^{a}$ Nighttime heartburn is defined as an event that occurred during the time after the subject consumed their evening meal until the time they got up the next morning to start daily activities
A daily diary card response of " 0 " for each parameter on $\geq 6$ of the subject's last 7 consecutive days in the study, allowing for 1 day with a maximum severity of " 2 "

No events reported during final 7 consecutive days of treatment

Number of days with heartburn during placebo run-in minus number of days with heartburn during final 7 consecutive days of treatment
Mean severity score for each parameter during placebo run-in minus mean severity score during final 7 consecutive days of treatment

Percentage of 24-h days without an incidence of each parameter over 2-week treatment period

Average number of events per day for each parameter during placebo run-in minus average number of events per day during final 7 consecutive days of treatment 
30 subjects would be randomized to each esomeprazole $20 \mathrm{mg}$ group and 15 subjects to placebo, with the goal of having $\sim 50$ subjects complete the study. Owing to the preliminary nature of this study, the sample size was not based on statistical calculations.

Logistic regression analyses using the PP population were performed with relief and complete resolution of $24-\mathrm{h}$ and nighttime heartburn (yes/no) on day 14 (dependent variables) and change in percentage of time with intragastric $\mathrm{pH}>4$ (independent variable), controlling for age, sex, and BMI. The analyses conducted with the FAS utilized a similar model, controlling for age, sex, and BMI; treatment was also included in this model. Summary measures included odds ratio (OR) for change in percentage of time with intragastric $\mathrm{pH}>4$ or change in median intragastric $\mathrm{pH}$ with $95 \%$ confidence interval (CI) of the OR. Boxplots of change in percentage of time with intragastric $\mathrm{pH}>4$ and change in median intragastric $\mathrm{pH}$ were constructed for subjects by status of relief and complete resolution of 24-h and nighttime heartburn.

Linear regressions were performed with improvement in the frequency and severity of 24-h and nighttime heartburn and average daily number of heartburn events at day 14 (dependent variables) and change in percentage of time with intragastric $\mathrm{pH}>4$ (independent variable), controlling for age, sex, and BMI. Similar models were used with change in median intragastric $\mathrm{pH}$ as the independent variable. The coefficient of change in percentage of time with intragastric $\mathrm{pH}>4$ or change in median intragastric $\mathrm{pH}$ was calculated along with associated $P$ values and 95\% CIs. SAS ${ }^{\circledR}$ software (version 9.4; SAS Institute; Cary, NC, USA) was used to perform all statistical analyses.

\section{RESULTS}

A total of 55 subjects were randomized to one of the three treatment arms; all 55 subjects were included in the FAS population (Fig. 2). The PP population included 39 subjects (16 subjects randomized to esomeprazole $20 \mathrm{mg}$ /esomeprazole $20 \mathrm{mg}, 18$ subjects randomized to esomeprazole $20 \mathrm{mg} /$ placebo, and 5 subjects randomized to placebo/placebo). Among those excluded from the PP population, most were not evaluable because they took rescue medication for $>2$ days during days 8-14 and thus $>2$ days of diary data were set to be missing during that period, violating the PP population's definition. Owing to the nature of this proof-of-concept study, the PP population was used for all endpoint analyses; only select analyses were performed on the FAS population. Results of some FAS analyses, which included 12 placebo subjects, are also reported. Demographic and baseline characteristics of the study population were similar among the three groups (Table 2).

\section{Relationship Between Intragastric pH and 24-h Heartburn Relief}

In the PP population $(n=39)$, the mean ( \pm standard deviation $[\mathrm{SD}]$ ) change from baseline in percentage of time with intragastric $\mathrm{pH}>4$ was $48.7 \%( \pm 29.7 \%)$. For the different subgroups, the mean change from baseline was $70.2 \%( \pm 23.1 \%)$ for esomeprazole $20 \mathrm{mg} /$ esomeprazole $20 \mathrm{mg}, \quad 42.8 \% \quad( \pm 18.7 \%)$ for esomeprazole $20 \mathrm{mg} /$ placebo, and $0.78 \%$ $( \pm 5.8 \%)$ for placebo/placebo. The mean (SD) change from baseline in percentage of time with intragastric $\mathrm{pH}>4$ for the portion of the PP population who achieved 24-h heartburn relief was $58.7 \%( \pm 26.4 \%)$ versus $41.0 \%( \pm 30.4 \%)$ for those who did not achieve 24-h heartburn relief.

A total of $17(43.6 \%)$ subjects in the PP population reported 24-h heartburn relief, including 7 (43.8\%) for esomeprazole $20 \mathrm{mg} /$ esomeprazole $20 \mathrm{mg}, \quad 10 \quad(55.6 \%)$ for esomeprazole $20 \mathrm{mg} /$ placebo, and $0(0.0 \%)$ for placebo/placebo. The remaining 22 (56.4\%) subjects did not report 24-h heartburn relief. For subjects reporting 24-h heartburn relief, the mean $( \pm \mathrm{SD})$ change from baseline in percentage of time with intragastric $\mathrm{pH}>4$ was $78.6 \%$ $( \pm 14.6 \%)$ and $44.7 \%( \pm 23.9 \%)$ for esomeprazole $20 \mathrm{mg}$ /esomeprazole $20 \mathrm{mg}$ and esomeprazole $20 \mathrm{mg} /$ placebo, respectively.

There was a significant correlation between change in percentage of time with intragastric 


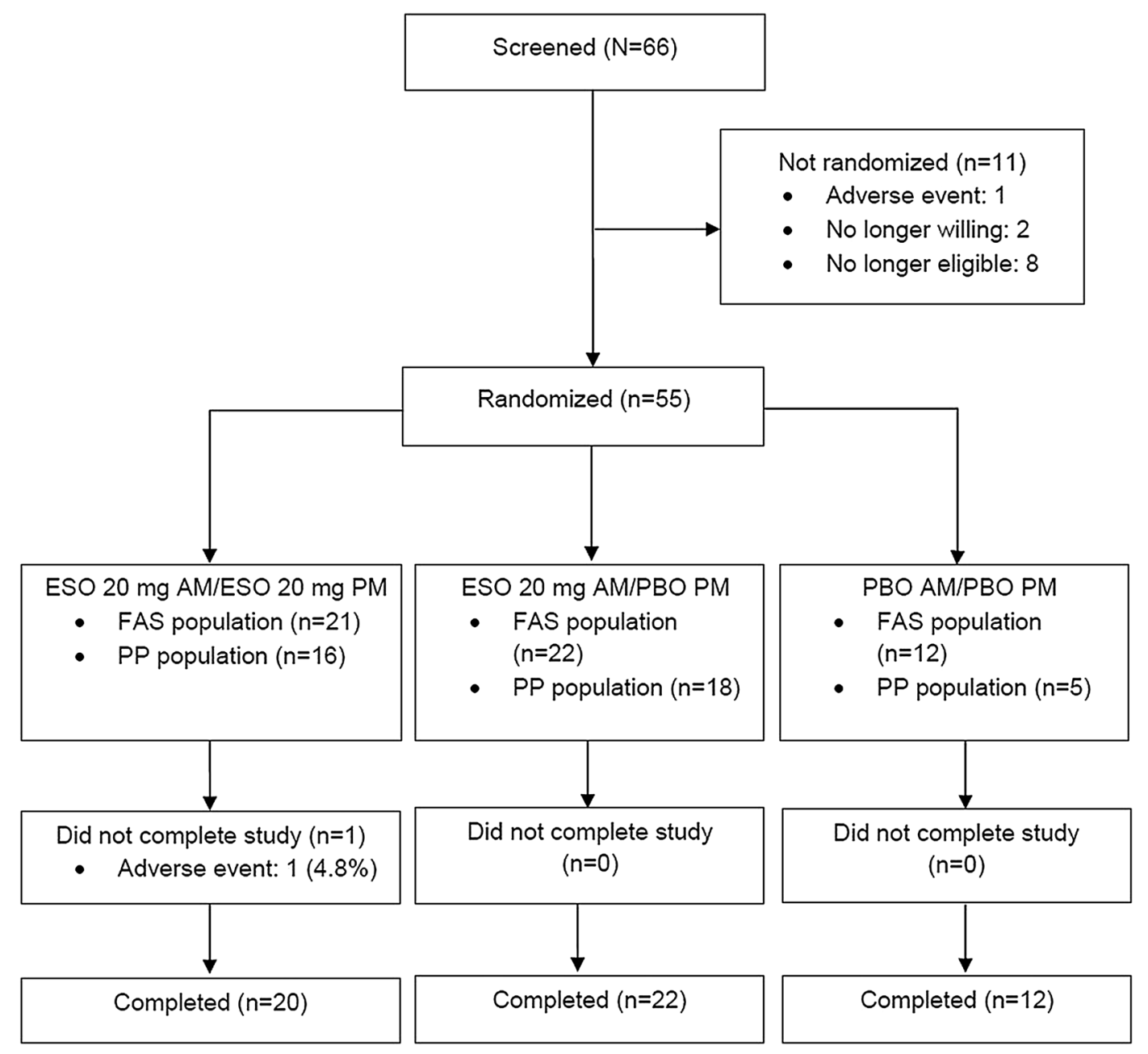

Fig. 2 Subject disposition. The per-protocol population included only evaluable subjects, defined as subjects who completed the 14-day treatment phase, underwent and provided valid data for day- $14 \mathrm{pH}$ monitoring and

$\mathrm{pH}>4$ and 24-h heartburn relief in the PP population, controlling for age, sex, and BMI [OR (95\% CI) 1.028 (1.001, 1.055); $P=0.0442$; Fig. 3]. A significant relationship was also observed between change in median intragastric $\mathrm{pH}$ and 24-h heartburn relief [OR (95\% CI) 1.629 (1.002, $2.650) ; P=0.0493]$. For the FAS population $(n=55)$, the OR for the relationship between 24-h heartburn relief and change from baseline in the percentage of time with intragastric $\mathrm{pH}>4$ [OR 1.027 (95\% CI 0.991, 1.065)] was directionally similar to the OR in the PP analyses but no longer significant $(P=0.1451)$. These completed $\geq 5$ days of diary entries on each of days -7 to -1 and days 8-14. ESO esomeprazole, FAS full analysis set, $\mathrm{PBO}$ placebo, PP per-protocol

analyses were adjusted for treatment in the model, which may have led to the loss of statistical significance compared with the PP analyses.

\section{Relationship Between Intragastric pH and Other Efficacy Parameters}

Complete 24-h heartburn resolution was significantly related to change in percentage of time with intragastric $\mathrm{pH}>4$, but not with change in median intragastric $\mathrm{pH}$ (Table 3; Fig. 4). Similar to the FAS analyses for heartburn relief, the association between complete 24-h 
Table 2 Demographic and baseline characteristics

\begin{tabular}{|c|c|c|c|c|}
\hline & $\begin{array}{l}\text { Total } \\
(N=55)\end{array}$ & $\begin{array}{l}\text { Esomeprazole } \\
20 \mathrm{mg} / \text { esomeprazole } \\
20 \mathrm{mg}(n=21)\end{array}$ & $\begin{array}{l}\text { Esomeprazole } \\
20 \mathrm{mg} / \text { placebo } \\
(n=22)\end{array}$ & $\begin{array}{l}\text { Placebo/placebo } \\
(n=12)\end{array}$ \\
\hline \multicolumn{5}{|l|}{ Sex, $n(\%)$} \\
\hline Female & $34(61.8)$ & $11(52.4)$ & $14(63.6)$ & $9(75.0)$ \\
\hline \multicolumn{5}{|l|}{ Age, years } \\
\hline Mean (SD) & $41.9(10.5)$ & $41.6(10.2)$ & $40.8(11.0)$ & $44.7(10.5)$ \\
\hline \multicolumn{5}{|l|}{ Race, $n$ (\%) } \\
\hline White & $47(85.5)$ & $17(81.0)$ & $22(100)$ & $8(66.7)$ \\
\hline Black & $4(7.3)$ & $1(4.8)$ & $0(0)$ & $3(25.0)$ \\
\hline Other & $4(7.3)$ & $3(14.3)$ & $0(0)$ & $1(8.3)$ \\
\hline \multicolumn{5}{|l|}{ Ethnicity, $n$ (\%) } \\
\hline Hispanic/Latino & $4(7.3)$ & $1(4.8)$ & $3(13.6)$ & $0(0)$ \\
\hline \multicolumn{5}{|l|}{ Weight, kg } \\
\hline Mean (SD) & $94.9(21.2)$ & $96.5(19.1)$ & $93.0(24.3)$ & $95.7(20.1)$ \\
\hline \multicolumn{5}{|l|}{ Height, cm } \\
\hline Mean (SD) & $170.6(10.4)$ & $171.0(10.8)$ & $171.3(11.2)$ & $168.7(8.4)$ \\
\hline \multicolumn{5}{|l|}{ BMI, $\mathrm{kg} / \mathrm{m}^{2}$} \\
\hline Mean (SD) & $32.5(6.2)$ & $32.9(5.0)$ & $31.5(6.8)$ & $33.7(7.2)$ \\
\hline
\end{tabular}

$B M I$ body mass index, $S D$ standard deviation

heartburn resolution and change in percentage of time with intragastric $\mathrm{pH}>4$ using the FAS population revealed a trend that was similar to PP analyses but nonsignificant [OR 1.043 (95\% CI 0.996, 1.091); $P=0.0737$. Using PP population data, there were also significant associations $(P<0.05)$ between both change in percentage of time with intragastric $\mathrm{pH}>4$ and median change in intragastric $\mathrm{pH}$ and observed improvement in 24-h heartburn severity, improvement in frequency and severity of nighttime heartburn, and change in percentage of 24-h days without epigastric pain (Table 3 ).

\section{Relationship Between Intragastric pH and QOLRAD}

After controlling for age, sex, BMI, and corresponding baseline score, significant correlations were observed between change in percentage of time with intragastric $\mathrm{pH}>4$ and improvement in the QOLRAD domain scores for emotional distress $(P=0.0381)$, food/drink problems $(P=0.0476)$, and physical/social functioning $(P=0.0222)$. There was no significant association between the percentage of time with intragastric $\mathrm{pH}>4$ and other QOLRAD domains. Physical/social functioning was the only parameter significantly associated with the change in median intragastric $\mathrm{pH}$ (Table 4). 


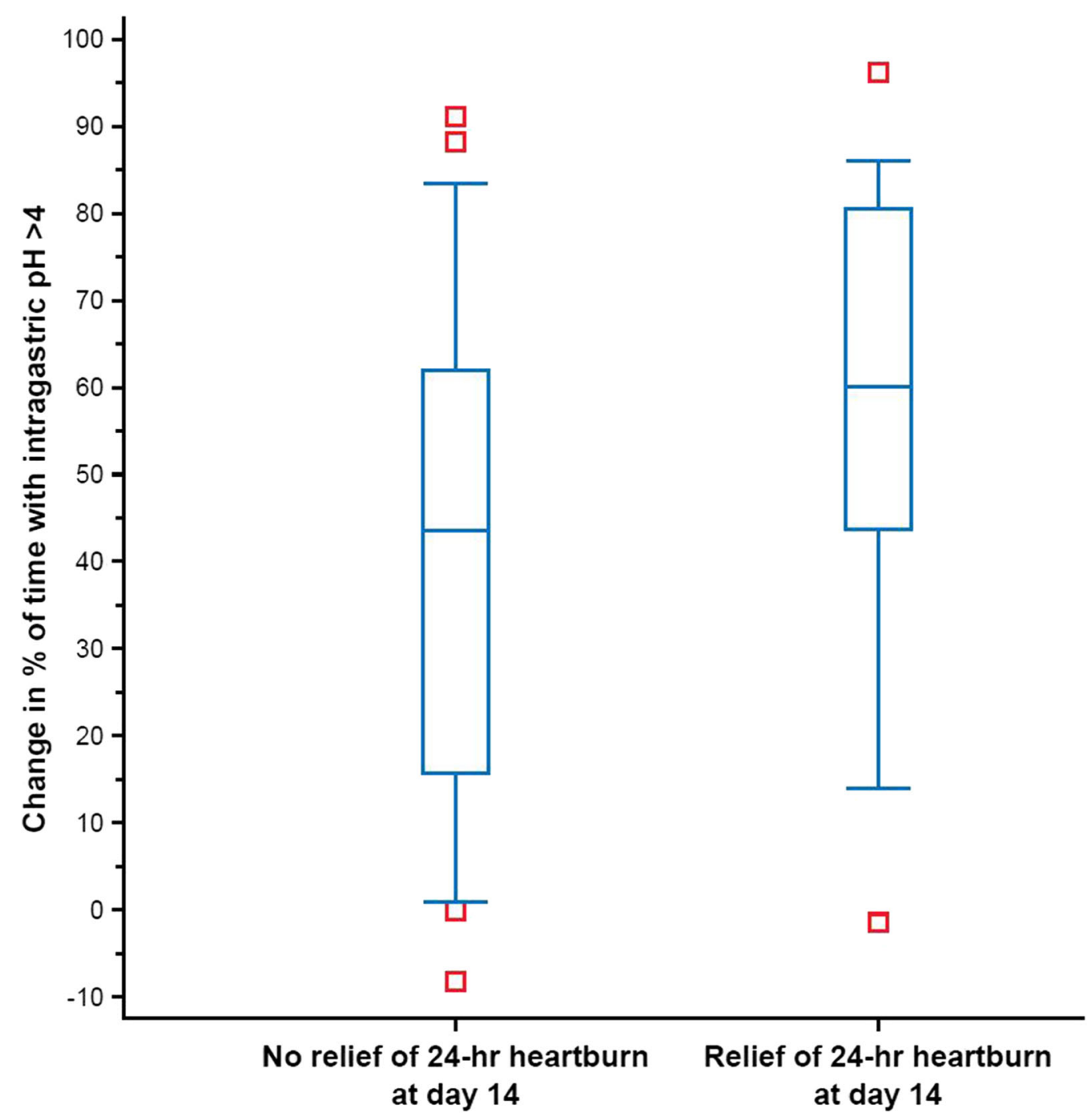

Fig. 3 Boxplot of change in percentage of time with intragastric $\mathrm{pH}>4$ for subjects with relief of 24-h heartburn vs. no relief of $24-h$ heartburn at day 14 , perprotocol population. Thirty-nine subjects are included in this plot for 24-h heartburn at day 14, among whom 17

\section{Relationship Between Change in Percentage of Time with Esophageal $\mathrm{pH}<4$ and Symptom Response}

The change in percentage of time with esophageal $\mathrm{pH}<4$ was significantly correlated with improvements in average daily number of heartburn events (coefficient 1.191; 95\% CI $0.688,1.693 ; \quad P<0.0001), \quad 24-\mathrm{h}$ heartburn $\mathrm{s}-$ had relief and 22 did not have relief. This boxplot provides median and the 25th/75th percentiles, with whisker tops at the 90th percentile and whisker bottoms at the 10th percentile. Data points that are outside the percentile range are represented with red squares

everity (coefficient 0.120; 95\% CI 0.029, 0.211; $P=0.0115$ ), and nighttime heartburn severity (coefficient $0.120 ; \quad 95 \%$ CI $0.036,0.205$; $P=0.0063)$. There were also significant correlations between change in percentage of time with esophageal $\mathrm{pH}<4$ and QOLRAD food/drink problems (coefficient 0.478; 95\% CI $0.031,0.924 ; P=0.0367$ ) and vitality (coefficient 0.212 ; $95 \%$ CI $0.018,0.407 ; P=0.0333$ ). 
Table 3 Relationship between intragastric $\mathrm{pH}$ and symptomatic outcomes, per-protocol population

\begin{tabular}{|c|c|c|}
\hline & $\begin{array}{l}\text { Change in percentage of } \\
\text { time with intragastric } \\
\mathrm{pH}>4\end{array}$ & Median intragastric $\mathrm{pH}$ \\
\hline \multicolumn{3}{|l|}{ 24-h heartburn } \\
\hline Relief, ${ }^{\mathrm{a}^{*}}$ & 1.028 (1.001, 1.055); 0.0442 & $1.629(1.002,2.650) ; 0.0493$ \\
\hline Complete resolution ${ }^{\mathrm{a}, *}$ & $1.034(1.003,1.065) ; 0.0301$ & $1.690(0.990,2.884) ; 0.0545$ \\
\hline \multicolumn{3}{|l|}{ Improvement $\mathrm{b}^{\mathrm{b}, *}$} \\
\hline Frequency & $\begin{array}{l}0.032(-0.002,0.065) \\
0.0627\end{array}$ & $0.475(-0.115,1.066) ; 0.1112$ \\
\hline Average number of daily events & $\begin{array}{l}0.109(-0.007,0.225) \\
0.0652\end{array}$ & $1.707(-0.350,3.764) ; 0.1009$ \\
\hline Severity & $0.026(0.010,0.042) ; 0.0029$ & $0.396(0.097,0.694) ; 0.0109$ \\
\hline Change in percentage of days without heartburn ${ }^{\mathrm{b}, *}$ & $\begin{array}{l}0.326(-0.091,0.744) \\
\quad 0.1214\end{array}$ & $\begin{array}{l}4.950(-2.426,12.326) \\
\quad 0.1815\end{array}$ \\
\hline \multicolumn{3}{|l|}{ Nighttime heartburn $^{c}$} \\
\hline Relief ${ }^{\mathrm{a},}{ }^{*}$ & 1.025 (1.000, 1.050); 0.0525 & $1.396(0.926,2.104) ; 0.1110$ \\
\hline Complete resolution ${ }^{\mathrm{a},{ }^{*}}$ & $1.010(0.988,1.033) ; 0.3715$ & $1.176(0.796,1.739) ; 0.4147$ \\
\hline \multicolumn{3}{|l|}{ Improvement $\mathrm{t}^{\mathrm{b}, *}$} \\
\hline Frequency & $0.038(0.009,0.067) ; 0.0123$ & 0.579 (0.060, 1.098); 0.0297 \\
\hline Severity & $0.021(0.005,0.037) ; 0.0128$ & $0.312(0.023,0.601) ; 0.0352$ \\
\hline \multicolumn{3}{|l|}{ Acid regurgitation } \\
\hline Relief $^{\mathrm{a}^{*}}$ & $1.013(0.989,1.039) ; 0.2887$ & $1.283(0.832,1.978) ; 0.2591$ \\
\hline Complete resolution ${ }^{\mathrm{a},}$ & $1.004(0.981,1.028) ; 0.7490$ & 1.059 (0.702, 1.599); 0.7833 \\
\hline $\begin{array}{l}\text { Change in percentage of days without acid } \\
\text { regurgitation } \text { b,** }^{\text {s.t. }}\end{array}$ & $\begin{array}{l}0.302(-0.078,0.681) \\
\quad 0.1155\end{array}$ & $\begin{array}{l}5.245(-1.406,11.896) \\
0.1183\end{array}$ \\
\hline \multicolumn{3}{|l|}{ Improvement $\mathrm{t}^{\mathrm{b}, *}$} \\
\hline Average number of daily events & $\begin{array}{l}0.005(-0.010,0.020) \\
0.5256\end{array}$ & $0.063(-0.202,0.329) ; 0.6301$ \\
\hline \multicolumn{3}{|l|}{ Epigastric pain } \\
\hline Relief $^{\mathrm{a}^{*}{ }^{*}}$ & $1.011(0.988,1.034) ; 0.3587$ & $1.250(0.834,1.875) ; 0.2797$ \\
\hline Complete resolution ${ }^{\mathrm{a}, *}$ & 1.007 (0.985, 1.030); 0.5309 & $1.113(0.755,1.640) ; 0.5900$ \\
\hline $\begin{array}{l}\text { Change in percentage of days without epigastric pain }{ }^{\mathrm{b}, * *} \\
\text { Improvement } \mathrm{t}^{\mathrm{b}, * *}\end{array}$ & $0.405(0.035,0.775) ; 0.0331$ & $6.563(0.012,13.113) ; 0.0496$ \\
\hline
\end{tabular}


Table 3 continued

\begin{tabular}{|c|c|c|}
\hline & $\begin{array}{l}\text { Change in percentage of } \\
\text { time with intragastric } \\
\mathrm{pH}>4\end{array}$ & Median intragastric $\mathrm{pH}$ \\
\hline Average number of daily events & $0(-0.011,0.012) ; 0.9332$ & $\begin{array}{l}-0.007(-0.204,0.190) ; \\
0.9402\end{array}$ \\
\hline
\end{tabular}

$B M I$ body mass index, $C I$ confidence interval, $O R$ odds ratio

Data are presented as ${ }^{*}\left[\mathrm{OR}\right.$ ( $95 \% \mathrm{CI}$ ); $P$ value] or ${ }^{* *}$ [Coefficient (95\% CI); $P$ value]

${ }^{a}$ Logistic regression model with each parameter at day 14 as dependent variable and change in percentage of time with intragastric $\mathrm{pH}>4$ or change in median intragastric $\mathrm{pH}$ as the independent variable, controlling for age, sex, and $\mathrm{BMI}$

b Linear regression model with each parameter at day 14 as dependent variable and change in percentage of time with intragastric $\mathrm{pH}>4$ or change in median intragastric $\mathrm{pH}$ as the independent variable, controlling for age, sex, and $\mathrm{BMI}$

c Nighttime heartburn is defined as an event that occurred during the time after the subject consumed their evening meal until the time they got up the next morning to start daily activities

\section{Relationship Between Change in Median Esophageal pH and Symptom Response}

Overall, the mean (SD) change in percentage of time with esophageal $\mathrm{pH}<4$ was $4.6 \%$ $( \pm 5.8 \%)$. The only outcome correlated with change in median esophageal $\mathrm{pH}$ was improvement in average number of daily heartburn events (coefficient - 11.268; 95\% CI $-21.356,-1.180 ; P=0.0297)$.

\section{Safety}

In the safety population, a total of 9 AEs were reported; 8 were determined to be treatment emergent [6 (10.9\%) subjects], and 1 (1.8\%) was not considered treatment emergent. One subject reported an $\mathrm{AE}$ of migraine resulting in permanent study medication discontinuation and study withdrawal. The most frequently reported $\mathrm{AE}$ was upper respiratory tract infection [3 (5.5\%)], reported by 2 subjects $(9.5 \%)$ with esomeprazole $20 \mathrm{mg}$ /esomeprazole $20 \mathrm{mg}$ and 1 subject $(4.5 \%)$ with esomeprazole $20 \mathrm{mg} /$ placebo. The next most common AE was gastroenteritis, which was reported by 2 subjects (3.6\%), both in the placebo/placebo group.

\section{DISCUSSION}

The established association between improvements in reflux symptoms following gastric acid suppression with a PPI has historically been based on statistical comparisons of means from study populations as a whole [7, 11, 13]. Analyzing the relationship between changes in acid suppression and symptom response using individual data demonstrated an increase in percentage of time with intragastric $\mathrm{pH}>4$ of $17.7 \%$, which provided greater heartburn relief compared with those not achieving this degree of change in $\mathrm{pH}$. A significant positive correlation was observed between both changes in percentage of time with intragastric $\mathrm{pH}>4$ and median intragastric $\mathrm{pH}$ with 24-h heartburn relief (both $P<0.05$ ). The observed significant relationship between the degree of change in time with intragastric $\mathrm{pH}>4$ and symptom relief suggests that individuals not achieving adequate acid control may benefit from a change in treatment regimen, such as increasing the dose of their acid-suppressive therapy. The relationship between the change in intragastric $\mathrm{pH}$ and symptom relief was clinically significant enough to warrant the use of empiric PPI treatment before performing other diagnostic investigations, including endoscopy. 


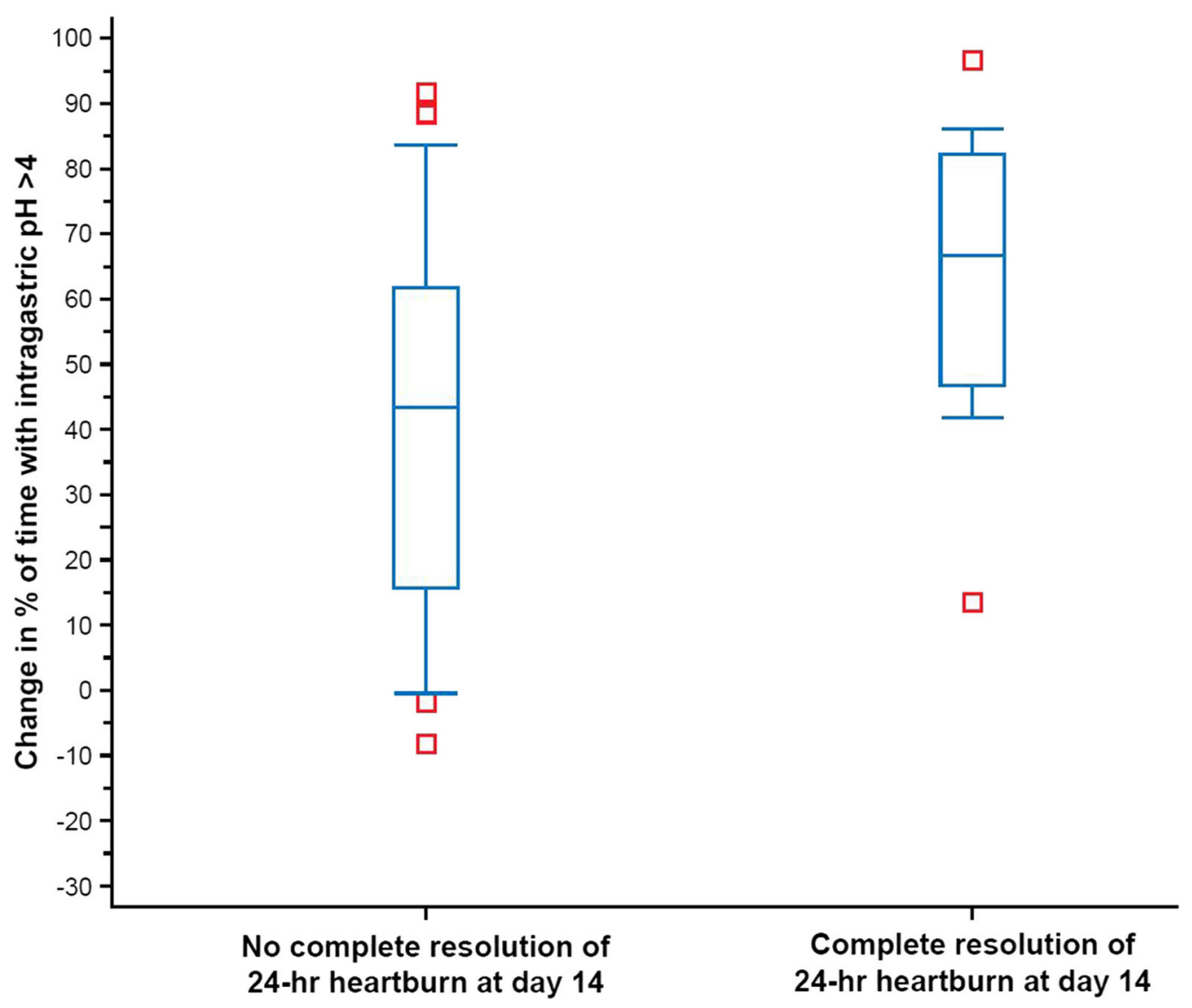

Fig. 4 Boxplot of change in percentage of time with intragastric $\mathrm{pH}>4$ for subjects with complete resolution of 24-h heartburn vs. no resolution of 24-h heartburn at day 14, per-protocol population. Thirty-nine subjects are included in this plot for 24-h heartburn at day 14 , including 13 with complete resolution and 26 without

To our knowledge only one previous study investigated this relationship in the context of healing EE [11]. Similar to the current results, Katz et al. reported significant correlations between controlling daytime and nighttime heartburn and acid regurgitation after 28 days of treatment and percentage of time with $\mathrm{pH}>4$ on day 5 [11]. However, those data were analyzed on a population level rather than individually, which could be important to consider for individuals not fully responding to PPI therapy. While this previous study demonstrated the efficacy of PPIs for reducing acid secretion using population-level data, individual degrees of change in intragastric $\mathrm{pH}$ and the relationship to treatment response have not complete resolution. Boxplot provides median and the 25 th/75th percentiles, with whisker tops at the 90th percentile and whisker bottoms at the 10th percentile. Data points that are outside the percentile range are represented with red squares

been assessed in a population that is likely to self-treat their symptoms in a way that is consistent with empiric PPI treatment for heartburn.

In the current study, we observed a significant association between complete heartburn resolution and percentage of time with intragastric $\mathrm{pH}>4$. The previous $\mathrm{EE}$ study only found a numerical (nonsignificant) association between these outcomes [11]. Our study also found a significant correlation between epigastric pain and percentage of time with intragastric $\mathrm{pH}>4$. A significant correlation was also observed between QOLRAD emotional distress, food/drink problems, and physical/social functioning and change in percentage of time with 
Table 4 Association between change in percentage of time with intragastric $\mathrm{pH}>4$ or median intragastric $\mathrm{pH}$ and improvement in QOLRAD domains at day 14, per-protocol population

\begin{tabular}{lll}
\hline Parameter & Coefficient $(\mathbf{9 5 \%}$ CI $)$ & $\boldsymbol{P}$ value \\
\hline Change in percentage of time with intragastric $\mathrm{pH}>4$ & & \\
Emotional distress & $0.074(0.004,0.144)$ & 0.0381 \\
Sleep disturbance & $0.053(-0.002,0.107)$ & 0.0602 \\
Food/drink problems & $0.086(0.001,0.170)$ & 0.0476 \\
Physical/social functioning & $0.053(0.008,0.098)$ & 0.0222 \\
Vitality & $0.036(-0.001,0.074)$ & 0.0578 \\
Change in median intragastric pH & & \\
Emotional distress & $1.009(-0.229,2.246)$ & 0.1066 \\
Sleep disturbance & $0.805(-0.172,1.782)$ & 0.1032 \\
Food/drink problems & $1.219(-0.301,2.740)$ & 0.1123 \\
Physical/social functioning & $0.813(0.009,1.616)$ & 0.0475 \\
Vitality & $0.560(-0.108,1.228)$ & 0.0976 \\
\hline
\end{tabular}

CI confidence interval, QOLRAD Quality of Life in Reflux and Dyspepsia

intragastric $\mathrm{pH}>4$. This is not unexpected, as a previous study observed a reduction in heartburn frequency and improved QOLRAD scores subsequent to potent acid suppression therapy, but that study did not evaluate these findings in relation to intragastric $\mathrm{pH}$ [14]. The current study demonstrated that individual changes in percentage of time with esophageal $\mathrm{pH}<4$ were correlated with improvements in heartburn frequency and severity, QOLRAD food/drink problems, and vitality. This is consistent with the observation that omeprazole effectively reduced both daytime and nighttime esophageal acid exposure in subjects experiencing EE healing [15].

The observed correlation between acid control and heartburn relief occurred in all subjects whether they received active treatment or placebo. These results confirm the wide variability in individual gastric acid secretion as a part of normal gastrointestinal physiology while supporting the observation that a greater decrease in gastric acid secretion is correlated with a greater likelihood of symptomatic improvement. Although there were changes in the amount of time with intragastric $\mathrm{pH}>4$ for both those who did and did not achieve relief, the difference between these groups was significant, which may represent an area of individual esophageal sensitivity. The substantial variability in $\mathrm{pH}$ measurements in the placebo group is a surprising and interesting finding and may help to explain high placebo response rates in these types of studies. This observation might have been missed if these data were not analyzed individually. The mean change from baseline in percentage of time with $\mathrm{pH}>4$ was $70.2 \%$ with esomeprazole $20 \mathrm{mg} /$ esomeprazole $20 \mathrm{mg}$ and $42.8 \%$ with esomeprazole $20 \mathrm{mg} /$ placebo compared with $0.78 \%$ with placebo/placebo. However, the number of subjects not experiencing heartburn relief despite substantial increases in time with intragastric $\mathrm{pH}>4$ may suggest that in certain individuals, heartburn can be attributed to factors other than reflux. As a result, identifying those individuals can help determine alternative treatment options beyond acid-suppressive therapy, which is consistent with use of empiric PPI therapy before performing other diagnostic 
studies, including endoscopic investigation, to diagnose those with heartburn related to acid reflux as well as those whose symptoms are from other causes. The observed dose-response relationship is expected and consistent with previous esomeprazole pharmacodynamic studies [16-19]. However, this apparent dose-response relationship did not translate proportionally to symptom control; there was a numerically higher rate of heartburn relief with esomeprazole once daily $(55.6 \%)$ compared with twice daily (43.8\%).

The primary limitation of this study was its preliminary nature. As proof-of-concept studies generally have relatively small populations, a more comprehensive study may confirm these results and establish a more sensitive assessment of the relationship between acid control and heartburn relief with different doses and dosing schedules. Future studies in this area may provide important insights into secondary treatment options for incomplete responders to OTC PPIs. In the current study, a larger proportion of subjects in the placebo group were removed from the PP population compared with the active treatment groups since subjects in the placebo group were more likely to require rescue medication, thereby violating the definition of the PP population. However, in analyses conducted with the total population, the direction of the relationship between 24-h heartburn relief and change from baseline in the percentage of time with intragastric $\mathrm{pH}>4$ was consistent with that of the PP population, despite no longer being statistically significant. We hope that our observations will improve the design of future protocols and that the information gathered from this study will be considered in future research in this area.

\section{CONCLUSION}

This pilot, proof-of-concept study demonstrated that the likelihood of individuals experiencing symptomatic relief improves relative to reductions in gastric acidity. This observation was valid across all subjects, including those receiving placebo. As expected, subjects in the esomeprazole cohorts experienced notable increases in symptomatic improvement, indicating that the endpoints are consistent with the pharmacotherapeutic effect of PPIs. Individuals experiencing a greater degree of change from baseline in percentage of the day with intragastric $\mathrm{pH}>4$ were more likely to experience reflux symptom relief or resolution than individuals with a lesser degree of change of time with intragastric $\mathrm{pH}>4$. Assessing individual-subject data helped interpret both the observed therapeutic failures with acidsuppressive therapy and placebo response in symptomatic heartburn. The data we present suggest that assessing changes in 24-h intragastric $\mathrm{pH}$ may be useful for deciding whether to increase the PPI dose or to explore alternative causes of heartburn in those not responding to empiric OTC PPI therapy. Evaluating the dose-response relationship with individual response levels would be useful in clinical management and can provide guidance for explaining the differential diagnosis of heartburn in individual patients. These results also support the use of empiric PPI treatment as a management strategy for frequent heartburn in the OTC setting.

\section{ACKNOWLEDGEMENTS}

The authors wish to acknowledge the clinicians who previously worked on this study, particularly James T. Angello, PharmD, and David Savastano, PhD. Their support was integral to the success of the study. We also thank the participants of the study.

Funding. This study was sponsored by Pfizer Consumer Healthcare, Madison, NJ, USA. The study sponsor also funded the article processing charges and Open Access fee. All authors had full access to all of the data in this study and take complete responsibility for the integrity of the data and accuracy of the data analysis.

Medical Writing and Editorial Assistance. Medical writing support was provided by Dennis Stancavish of Peloton Advantage, LLC, and was funded by Pfizer. 
Authorship. All named authors meet the International Committee of Medical Journal Editors (ICMJE) criteria for authorship for this article, take responsibility for the integrity of the work as a whole, and have given their approval for this version to be published.

Disclosures. Philip B. Miner, Jr has served as a consultant, advisory board member, and principal investigator for Pfizer. David A. Johnson has served as a consultant to Pfizer and Proctor \& Gamble and an advisory board member for Medscape/WebMD. Philip O. Katz is a consultant for Torax Medical. Jing $\mathrm{Li}$ is an employee of Pfizer Consumer Healthcare. Sergio C. Gatoulis is an employee of and owns stock in Pfizer. Charles Pollack was an employee of Pfizer Consumer Healthcare at the time of the study. Charles Pollack is now an employee of Avrio Health.

Compliance with Ethics Guidelines. This study complied with the ethical principles of the Declaration of Helsinki and all International Conference for Harmonisation Good Clinical Practice Guidelines. The final protocol, any amendments, and informed consent forms were reviewed and approved on December 9, 2015, by an independent institutional review board at the investigational center (Oklahoma Foundation for Digestive Research; Oklahoma City, OK, USA). Informed consent was obtained from all individual participants included in the study.

Data Availability. Upon request, and subject to certain criteria, conditions and exceptions, see https://www.pfizer.com/science/ clinical-trials/trial-data-and-results for more information, Pfizer will provide access to individual de-identified participant data from Pfizer-sponsored global interventional clinical studies conducted for medicines, vaccines, and medical devices (1) for indications that have been approved in the USA and/or EU or (2) in programs that have been terminated (i.e., development for all indications has been discontinued). Pfizer will also consider requests for the protocol, data dictionary, and statistical analysis plan. Data may be requested from Pfizer trials 24 months after study completion. The de- identified participant data will be made available to researchers whose proposals meet the research criteria and other conditions, and for which an exception does not apply, via a secure portal. To gain access, data requestors must enter into a data access agreement with Pfizer.

Open Access. This article is distributed under the terms of the Creative Commons Attribution-NonCommercial 4.0 International License (http://creativecommons.org/licenses/ by-nc/4.0/), which permits any noncommercial use, distribution, and reproduction in any medium, provided you give appropriate credit to the original author(s) and the source, provide a link to the Creative Commons license, and indicate if changes were made.

\section{REFERENCES}

1. Savarino V, Di Mario F, Scarpignato C. Proton pump inhibitors in GORD: an overview of their pharmacology, efficacy and safety. Pharmacol Res. 2009;59(3):135-53.

2. Katz PO, Gerson LB, Vela MF. Guidelines for the diagnosis and management of gastroesophageal reflux disease. Am J Gastroenterol. 2013;108(3):308-28.

3. Fiorucci S, Santucci L, Chiucchiu S, Morelli A. Gastric acidity and gastroesophageal reflux patterns in patients with esophagitis. Gastroenterology. 1992;103(3):855-61.

4. Rohss K, Hasselgren G, Hedenstrom H. Effect of esomeprazole $40 \mathrm{mg}$ vs omeprazole $40 \mathrm{mg}$ on 24-hour intragastric $\mathrm{pH}$ in patients with symptoms of gastroesophageal reflux disease. Dig Dis Sci. 2002;47(5):954-8.

5. Franco MT, Salvia G, Terrin G, et al. Lansoprazole in the treatment of gastro-oesophageal reflux disease in childhood. Dig Liver Dis. 2000;32(8):660-6.

6. Katz PO, Hatlebakk JG, Castell DO. Gastric acidity and acid breakthrough with twice-daily omeprazole or lansoprazole. Aliment Pharmacol Ther. 2000;14(6):709-14.

7. Bell NJ, Burget D, Howden CW, Wilkinson J, Hunt $\mathrm{RH}$. Appropriate acid suppression for the management of gastro-oesophageal reflux disease. Digestion. 1992;51(Suppl 1):59-67. 
8. Labenz J, Tillenburg B, Peitz U, et al. Efficacy of omeprazole one year after cure of Helicobacter pylori infection in duodenal ulcer patients. Am J Gastroenterol. 1997;92(4):576-81.

9. Sachs G, Shin JM, Briving C, Wallmark B, Hersey S. The pharmacology of the gastric acid pump: the $\mathrm{H}+, \mathrm{K}+$ ATPase. Annu Rev Pharmacol Toxicol. 1995;35:277-305.

10. Andersson T, Rohss K, Bredberg E, Hassan-Alin M. Pharmacokinetics and pharmacodynamics of esomeprazole, the S-isomer of omeprazole. Aliment Pharmacol Ther. 2001;15(10):1563-9.

11. Katz PO, Ginsberg GG, Hoyle PE, Sostek MB, Monyak JT, Silberg DG. Relationship between intragastric acid control and healing status in the treatment of moderate to severe erosive oesophagitis. Aliment Pharmacol Ther. 2007;25(5):617-28.

12. Wiklund I, Junghard O, Grace E, et al. Quality of life in reflux and dyspepsia. Development and psychometric documentation of a disease-specific questionnaire (QOLRAD) (abstract G0199). Gastroenterology. 1998;114(suppl 1):A49.

13. Johnson DA, Katz PO, Levine D, et al. Prevention of relapse of healed reflux esophagitis is related to the duration of intragastric $\mathrm{pH}>4$. J Clin Gastroenterol. 2010;44(7):475-8.

14. Kahrilas PJ, Jonsson A, Denison H, Wernersson B, Hughes N, Howden CW. Impact of regurgitation on health-related quality of life in gastro-oesophageal reflux disease before and after short-term potent acid suppression therapy. Gut. 2014;63(5):720-6.

15. Dehn TC, Shepherd HA, Colin-Jones D, Kettlewell MG, Carroll NJ. Double blind comparison of omeprazole (40 mg od) versus cimetidine (400 mg qd) in the treatment of symptomatic erosive reflux oesophagitis, assessed endoscopically, histologically and by $24 \mathrm{~h} \mathrm{pH}$ monitoring. Gut. 1990;31(5):509-13.

16. Lind T, Rydberg L, Kyleback A, et al. Esomeprazole provides improved acid control vs. omeprazole in patients with symptoms of gastro-oesophageal reflux disease. Aliment Pharmacol Ther. 2000;14(7):861-7.

17. Rohss K, Wilder-Smith C, Naucler E, Jansson L. Esomeprazole $20 \mathrm{mg}$ provides more effective intragastric acid control than maintenance-dose rabeprazole, lansoprazole or pantoprazole in healthy volunteers. Clin Drug Investig. 2004;24(1):1-7.

18. Wilder-Smith C, Backlund A, Eckerwall G, Lind T, Fjellman M, Rohss K. Effect of increasing esomeprazole and pantoprazole doses on acid control in patients with symptoms of gastro-oesophageal reflux disease: a randomized, dose-response study. Clin Drug Investig. 2008;28(6):333-43.

19. Wilder-Smith C, Lind T, Lundin C, Naucler E, Nilsson-Pieschl C, Rohss K. Acid control with esomeprazole and lansoprazole: a comparative dose-response study. Scand J Gastroenterol. 2007;42(2):157-64. 